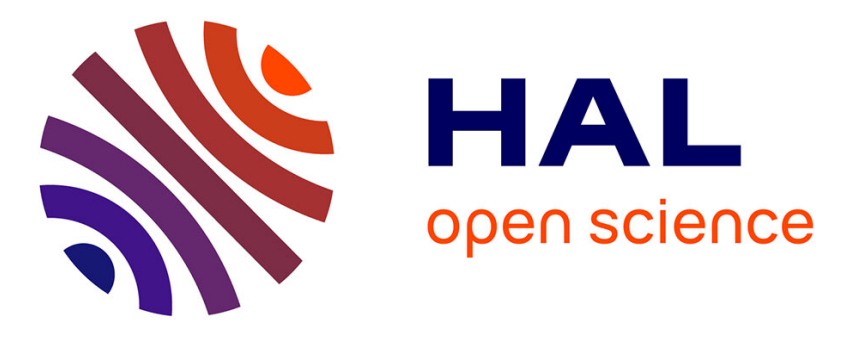

\title{
Judgment Aggregation with Abstentions under Voters' Hierarchy
}

\author{
Guifei Jiang, Dongmo Zhang, Laurent Perrussel
}

\section{To cite this version:}

Guifei Jiang, Dongmo Zhang, Laurent Perrussel. Judgment Aggregation with Abstentions under Voters' Hierarchy. 17th International Conference Pacific Rim international Conference on MultiAgents (PRIMA 2014), Dec 2014, Gold Coast, QLD, Australia. pp. 341-356. hal-01316837

\section{HAL Id: hal-01316837 \\ https://hal.science/hal-01316837}

Submitted on 17 May 2016

HAL is a multi-disciplinary open access archive for the deposit and dissemination of scientific research documents, whether they are published or not. The documents may come from teaching and research institutions in France or abroad, or from public or private research centers.
L'archive ouverte pluridisciplinaire HAL, est destinée au dépôt et à la diffusion de documents scientifiques de niveau recherche, publiés ou non, émanant des établissements d'enseignement et de recherche français ou étrangers, des laboratoires publics ou privés. 


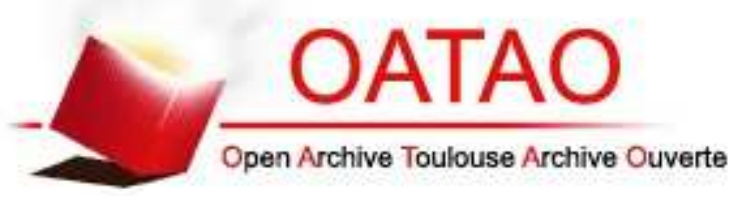

\section{Open Archive TOULOUSE Archive Ouverte (OATAO)}

OATAO is an open access repository that collects the work of Toulouse researchers and makes it freely available over the web where possible.

This is an author-deposited version published in : http://oatao.univ-toulouse.fr/ Eprints ID : 15446

The contribution was presented at :

http://www.prima2014.org

Official URL: http://dx.doi.org/10.1007/978-3-319-13191-7_28

To cite this version : Jiang, Guifei and Zhang, Dongmo and Perrussel, Laurent Judgment Aggregation with Abstentions under Voters' Hierarchy. (2014) In: 17th International Conference Pacific Rim international Conference on Multi-Agents (PRIMA 2014), 1 December 2014 - 5 December 2014 (Gold Coast, QLD, Australia).

Any correspondence concerning this service should be sent to the repository administrator: staff-oatao@listes-diff.inp-toulouse.fr 


\title{
Judgment Aggregation with Abstentions under Voters' Hierarchy
}

\author{
Guifei Jiang $^{1,2}$, Dongmo Zhang ${ }^{1}$, and Laurent Perrussel ${ }^{2}$ \\ 1 AIRG, University of Western Sydney, Australia \\ ${ }^{2}$ IRIT, University of Toulouse, France
}

\begin{abstract}
Similar to Arrow's impossibility theorem for preference aggregation, judgment aggregation has also an intrinsic impossibility for generating consistent group judgment from individual judgments. Removing some of the pre-assumed conditions would mitigate the problem but may still lead to too restrictive solutions. It was proved that if completeness is removed but other plausible conditions are kept, the only possible aggregation functions are oligarchic, which means that the group judgment is purely determined by a certain subset of participating judges. Instead of further challenging the other conditions, this paper investigates how the judgment from each individual judge affects the group judgment in an oligarchic environment. We explore a set of intuitively demanded conditions under abstentions and design a feasible judgment aggregation rule based on the agents' hierarchy. We show this proposed aggregation rule satisfies the desirable conditions. More importantly, this rule is oligarchic with respect to a subset of agenda instead of the whole agenda due to its literal-based characteristics.
\end{abstract}

\section{Introduction}

Judgment aggregation is an interdisciplinary research topic in economics, philosophy, political science, law and recently in computer science [1-5]. It deals with the problem of how a group judgment on certain issues, represented in logical propositions, can be formed based on individuals' judgments on the same issues. Although most of voting rules for social choice, such as majority, two-thirds majority or unanimity, are applicable to judgment aggregation, their behaviour can be significantly different due to possible logical links among the propositions on which a collective decision has to be made. A well-known example is the so-called doctrinal paradox [6], which shows that the majority rule fails to guarantee consistent group judgments.

Suppose a court consisting of three judges has to reach a verdict in a breachof-contract case. There are three propositions on which the court is required to make judgments:

$p$ : The defendant was contractually obliged not to do a particular action.

$q$ : The defendant did that action.

$r$ : The defendant is liable for breach of contract. 
According to legal doctrine, propositions $p$ and $q$ are jointly necessary and sufficient for proposition $r$, that is $p \wedge q \leftrightarrow r$. Now the three judges' judgments on the propositions are showed in Table 1.

Table 1. A doctrinal paradox

\begin{tabular}{|r|r|r|r|}
\hline & $p$ & $q$ & $r$ \\
\hline Judge 1 & $\mathrm{T}$ & $\mathrm{T}$ & $\mathrm{T}$ \\
Judge 2 & $\mathrm{T}$ & $\mathrm{F}$ & $\mathrm{F}$ \\
Judge 3 & $\mathrm{F}$ & $\mathrm{T}$ & $\mathrm{F}$ \\
\hline Maj & $\mathrm{T}$ & $\mathrm{T}$ & $\mathrm{F}$ \\
\hline
\end{tabular}

If the three judges take a majority vote on proposition $r$ which is regarded as the conclusion, the outcome is its rejection: a 'not liable' verdict. But if they take majority votes on each of $p$ and $q$ instead, then $p$ and $q$ are accepted and hence by the legal doctrine, $r$ should be accepted as well: a 'liable' verdict. This specifically displays that the set of propositions $\{p, q, \neg r\}$ which is accepted by a majority is logically inconsistent relative to the constraint $p \wedge q \leftrightarrow r$. The problem generalizes well beyond this example and does not depend on the presence of any legal doctrine or exogenous constraints [7].

To illustrate a more general problem, consider any set of propositions with logical connections. Suppose a three-member committee has to make group judgments (acceptance/rejection) on three logically interconnected propositions:

$p$ : We can afford a budget deficit.

$p \rightarrow q$ : If we can afford a budget deficit, then we should spend more money on education.

$q$ : We should spend more money on education.

The individual judgments on given propositions for each member are shown in Table 2.

Table 2. A discursive dilemma

\begin{tabular}{|r|c|c|c|}
\hline & $p$ & $p \rightarrow q$ & $q$ \\
\hline 1 & $\mathrm{~T}$ & $\mathrm{~T}$ & $\mathrm{~T}$ \\
2 & $\mathrm{~T}$ & $\mathrm{~F}$ & $\mathrm{~F}$ \\
3 & $\mathrm{~F}$ & $\mathrm{~T}$ & $\mathrm{~F}$ \\
\hline Maj & $\mathrm{T}$ & $\mathrm{T}$ & $\mathrm{F}$ \\
\hline
\end{tabular}

Then each individual holds consistent judgments on the three propositions, and yet there are majorities for $p, p \rightarrow q$ and $\neg q$, a logically inconsistent set of propositions. The first is the demand that in aggregating judgment a group should be responsive to the views of members on each of judgments involved. The second is the demand that in aggregating judgment a group should reach a 
collective set of judgments that is itself rational. The paradox shows that the two demands are sometimes in conflict, so that a group that tries to aggregate judgment faces a dilemma. The fact that majority voting may generate inconsistent group judgments is called the discursive dilemma $[3,8,9]$.

Naturally, the observation that majority voting may fail to produce consistent group judgments raises several questions. In particular, there are two fundamental questions: First, how general is the problem? Is it restricted to majority voting, or does it extend to other decision methods? Secondly, does it occur only in special situations, such as the breach-of-contract case, or does it arise more generally?

In response to these questions, a growing literature on the impossibility of consistent judgment aggregation under various conditions springs up. List and Pettit showed an impossibility result, similar to Arrow's impossibility theorem [10], that no aggregation rule can generate consistent group judgments if we require the rule to satisfy a set of "plausible" conditions [11]. However, such an impossibility result did not discourage the investigation of the possibility of judgment aggregation. None of the conditions on either aggregation rules or decision problems, is indefectible. By weakening or varying these conditions, a growing body of literature on judgment aggregation has emerged in recent years [12-16].

Among all the plausible conditions that lead to impossibility results on judgment aggregation, completeness as one of the rationality requirements has received criticism of being overly demanding in many real-world situations, where an individual may abstain on a decision issue, and the group judgment on some issue may be undetermined. In fact, if we give up completeness, we are able to circumvent impossibility [11, 17-20]. Among them, Gärdenfors has proved a representation theorem for judgment aggregation without completeness, which shows that under certain fairly natural conditions, the only possible aggregation rules are oligarchic. Dietrich and List (2008) have strengthened Gärdenfors' results and showed that by giving up completeness in favor of deductive closure, oligarchies instead of dictatorships are obtained. However, this by no means is a negative result. In fact, our previous work [21] demonstrates that with abstentions, oligarchic aggregation is no longer a single level determination but can also be a multiple-level democracy, which partially explains its pervasiveness in the real world.

Since in our society the hierarchy is one of the most basic organization forms and a hierarchical group may give individual members or subgroups the priority to determine the group judgments on certain propositions. However, such kind of expert rights has been rarely investigated in the current literature [22], let alone proposing a specific judgment aggregation rule to formally display how the hierarchical groups generate the group judgments. In [21] we deal with this issue by proposing a quasi-lexicographic judgment aggregation rule which works well over the limited agenda. It mainly focuses on the following two questions: How does the hierarchical group generate the group judgments? How can the non-oligarchs have the power to make the collective decision in an oligarchic environment? 
In this paper, we continue this line of research and investigate the question of whether we can generate consistent group judgments when giving different agents different weights depending on their hierarchy on the propositions in question. We focus on judgment aggregation with abstentions under voter's hierarchy. Our contributions can be summarized in the following: First, we amend the "plausible" conditions in [21], which allows us to extend the set of propositions on which the quasi-lexicographic rule works well from a set of literals to logically interconnected formulas; Secondly, we propose a feasible literal-based aggregation rule for judgment aggregation with abstentions under voter's hierarchy, and show that it is neither dictatorial nor oligarchic over the whole set of agenda; Last but not least, to some extent, we circumvent the impossibility result in [23] by removing completeness from the requirements of both individual and collective levels.

The rest of this paper is structured as follows: In Section 2, we introduce the formal model of judgment aggregation with abstentions based on the formalisms in $[11,24]$. In section 3 , we list the conditions that sound natural in the context of abstentions and compare them with their counterparts in [19-21]. In Section 4, we propose a literal-based lexicographic judgment aggregation procedure and investigate its properties. In the last section, we conclude the paper with a discussion of further work.

\section{The Model of Judgment Aggregation with Abstentions}

We consider a finite set of individuals $N=\{1,2, \ldots, n\}$ with $|N| \geq 2$. They are faced with a decision problem that requires group judgments on logically interconnected propositions represented by a logical language $\mathcal{L}$ with a set $\Phi_{0}$ of atomic propositions and logical connectives $\{\neg, \vee, \wedge, \leftarrow, \leftrightarrow\}$. We assume that the underlying logic of the logical language is the classical propositional logic with standard syntax and semantics. The set of literals which are either propositional variables or negations of propositional variables, is denoted by $\mathcal{P}$, i.e., $\mathcal{P}=$ $\left\{p, \neg p \mid p \in \Phi_{0}\right\}$.

Given a decision-making problem, the set of propositions on which judgments are to be made is called the agenda. Formally, the agenda is a finite non-empty subset $X \subseteq \mathcal{L}$ that is closed under negation, i.e., if $\varphi \in X$, then $\neg \varphi \in X$, and under propositional variables, i.e., for all $\varphi \in \mathcal{L}$, if $\varphi \in X$, then for all $p \in \Phi_{0}$ occur in $\varphi, p \in X$. Let $X_{0}=X \cap \mathcal{P}$ be the set of literals included in the agenda. Consider the doctrinal paradox in Introduction as an example. In that situation, the agenda $X$ is $\{p, q, p \wedge q, \neg p, \neg q, \neg(p \wedge q)\}$, and the set $X_{0}$ of literals in the agenda is $\{p, q, \neg p, \neg q\}$. Similar to [18], we assume that double negations in the agenda cancel each other. That is $X=\left\{\varphi, \neg \varphi: \varphi \in X^{*}\right\}$ where $X^{*} \subseteq \mathcal{L}$ is a set of unnegated propositions.

We represent each individual judgment set as a subset of the agenda, which represents all the propositions that this individual accepts. The individual $i$ 's judgment set is denoted by $\Phi_{i}$, which is a subset of $X$. As we have mentioned in the previous section, we will not assume that each individual's judgment set 
must be complete. For each proposition $\varphi \in X$, it may happen that $\varphi \notin \Phi_{i}$ and $\neg \varphi \notin \Phi_{i}$. In this case, we say that individual $i$ abstains from making a judgment on $\varphi$, denoted by $\varphi \# \Phi_{i}$. In other words, $\varphi \# \Phi_{i}$ if and only if $\varphi \notin \Phi_{i}$ and $\neg \varphi \notin \Phi_{i}$. We assume that each individual's judgment satisfies the following conditions:

(Individual Logical Closure). For every $\varphi \in X$, if $\Phi_{i} \models \varphi$, then $\varphi \in \Phi_{i}$.

(Individual Consistency). For every $\varphi \in X$, if $\varphi \in \Phi_{i}$, then $\neg \varphi \notin \Phi_{i}$.

The first condition requires that each individual judgment set is logically closed, that is for any $\varphi$ in the agenda, if it is a logical consequence of an individual judgment set, then the individual accepts $\varphi$. The second condition specifies that each individual judgment set must be logical consistence, i.e., an individual cannot accept both $\varphi$ and $\neg \varphi$ for every proposition $\varphi$ in the agenda. Given each individual's judgment set $\Phi_{i}$, the vector $\left(\Phi_{i}\right)_{i \in N}$ is called a profile. For instance, the individual judgment set of each judge in the doctrinal paradox is as follows: $\Phi_{1}=\{p, q, p \wedge q\} ; \Phi_{2}=\{p, \neg q, \neg(p \wedge q)\} ; \Phi_{3}=\{\neg p, q, \neg(p \wedge q)\}$. They compose a profile.

Finally, an (judgment) aggregation rule is a function $F$ that assigns to each admissible profile $\left(\Phi_{i}\right)_{i \in N}$ a single group judgment set $\Phi \subseteq X$, where $\varphi \in \Phi$ means that the group accepts $\varphi$. The set of admissible profiles is called the domain of $F$, denoted as $\operatorname{Dom}(F)$. Note that we do not require the group judgments to be complete, which means that a group can also abstain from making a judgment on a proposition.

\section{Conditions on Aggregation Rules}

We now turn to investigating the conditions which are desirable to be put on an aggregation rule in terms of abstentions. Let $F$ be an aggregation function. We first consider the following three conditions:

Universal Domain (UD). The domain of $F$ includes all logically closed and consistent profiles $\left(\Phi_{i}\right)_{i \in N}$.

Collective Rationality (CR). For all $\varphi \in X$ and for all $\left(\Phi_{i}\right)_{i \in N} \in \operatorname{Dom}(F)$ $F\left(\left(\Phi_{i}\right)_{i \in N}\right) \models \varphi$ implies $\varphi \in F\left(\left(\Phi_{i}\right)_{i \in N}\right)$, and $\varphi \in F\left(\left(\Phi_{i}\right)_{i \in N}\right)$ implies $\neg \varphi \notin$ $F\left(\left(\Phi_{i}\right)_{i \in N}\right)$. This requires the collective judgment set is logical closed and consistent.

Non-dictatorship (ND). There is no $x \in N$ such that for all $\left\{\Phi_{i}\right\}_{i \in N} \in$ $\operatorname{Dom}(F), F\left(\left\{\Phi_{i}\right\}_{i \in N}\right)=\Phi_{x}$. This is a basic democratic requirement: no single individual should always determine the group judgment set.

The next condition is the counterpart of Unanimity with Abstentions in [21] which is restricted to literals.

Literal Unanimity with Abstentions (LU). For every $\alpha \in \mathcal{P}$, if there is some $V \subseteq N$ such that $V \neq \emptyset, \forall i \in V . \alpha \in \Phi_{i}$ and $\forall j \in N \backslash V . \alpha \# \Phi_{j}$, then $\alpha \in$ $F\left(\left(\Phi_{i}\right)_{i \in N}\right)$. Intuitively, if a set of individuals agrees on a certain judgment on a literal $\alpha$ while all the others abstain from $\alpha$, then this condition requires that $F\left(\left(\Phi_{i}\right)_{i \in N}\right)$ should accept $\alpha$ as well. 
As we will see in the following example, this new condition plays a crucial role in extending the agenda set from a set of literals to a set of logically interconnected formulas without generating inconsistent aggregate results. On the other hand, it is neither an extension nor a restriction of Unanimity without abstentions, also called Pareto optimality in [20] and Paretian condition in [19], which is described as follows:

For every $\varphi \in X$, if $\varphi \in \Phi_{i}$ for every $i \in N$, then $\varphi \in F\left(\left(\Phi_{i}\right)_{i \in N}\right)$.

The following proposition says that non-dictatorship can be derived from LU.

Proposition 1. Every judgment aggregation rule satisfying literal unanimity with abstentions is non-dictatorial.

Proof. Assume that $F$ is dictatorial in some individual $a \in N$, then $N /\{a\} \neq \emptyset$. Take $\alpha \in X_{0}$ and define $\alpha \# \Phi_{a}$ and $\alpha \in \Phi_{x}$ for every $x \in N /\{a\}$. By literal unanimity with abstentions, $\alpha \in F\left(\left(\Phi_{i}\right)_{i \in N}\right)$, then $F\left(\left(\Phi_{i}\right)_{i \in N}\right) \neq \Phi_{a}$, contradiction.

The following independence condition requires that the group judgment on each literal should depend only on individual judgments on that literal, which is a counterpart of Arrow's "independence of irrelevant alternative" [25]. With abstentions, this condition has two different versions:

Strong Literal Independence $\left(\mathbf{L I}^{s}\right)$. For every $\alpha \in \mathcal{P}$ and every profiles $\left(\Phi_{i}\right)_{i \in N},\left(\Phi_{i}^{\prime}\right)_{i \in N} \in \operatorname{Dom}(F)$, if $\alpha \in \Phi_{i} \leftrightarrow \alpha \in \Phi_{i}^{\prime}$ for every $i \in N$, then $\alpha \in F\left(\left(\Phi_{i}\right)_{i \in N}\right) \leftrightarrow \alpha \in F\left(\left(\Phi_{i}^{\prime}\right)_{i \in N}\right)$.

Weak Literal Independence (LI). For every $\alpha \in \mathcal{P}$ and every profiles $\left(\Phi_{i}\right)_{i \in N},\left(\Phi_{i}^{\prime}\right)_{i \in N} \in \operatorname{Dom}(F)$, if $\alpha \in \Phi_{i} \leftrightarrow \alpha \in \Phi_{i}^{\prime}$ and $\neg \alpha \in \Phi_{i} \leftrightarrow \neg \alpha \in \Phi_{i}^{\prime}$ for every $i \in N$, then $\alpha \in F\left(\left(\Phi_{i}\right)_{i \in N}\right) \leftrightarrow \alpha \in F\left(\left(\Phi_{i}^{\prime}\right)_{i \in N}\right)$.

Note that these two versions are the same if we assume individual completeness. However, once we allow abstentions, these two versions become different and the strong version is intuitively too strong to be acceptable. Since in the profile even if all judges who abstain on $\alpha$ turn to rejecting $\alpha$, no matter how big portion of these judges is, the strong version requires the group judgment on $\alpha$ to be the same so long as the same set of judges accepts $\alpha$. The weak version solves the problem.

Similar conditions have been also discussed in the literature. It is not hard to see that the strong (weak) literal Independence condition strengthens the independence of irrelevant propositional alternatives condition in [23] to the set of literals under the provision of abstentions, while the weak independence condition amounts to reserving the independent of irrelevant alternatives condition in [19] to literals.

The following two conditions are two versions of the counterpart of the neutrality condition, which requires that literals should be treated in an even-handed way by the aggregation function. 
Strong Literal Neutrality $\left(\mathbf{L N}^{s}\right)$. For every $\alpha, \beta \in \mathcal{P}$ and every profile $\left(\Phi_{i}\right)_{i \in N}$

$\in \operatorname{Dom}(F)$, if $\alpha \in \Phi_{i} \leftrightarrow \beta \in \Phi_{i}$ for every $i \in N$, then $\alpha \in F\left(\left(\Phi_{i}\right)_{i \in N}\right) \leftrightarrow \beta \in$ $F\left(\left(\Phi_{i}\right)_{i \in N}\right)$.

Weak Literal Neutrality (LN). For every $\alpha, \beta \in \mathcal{P}$ and every profile $\left(\Phi_{i}\right)_{i \in N}$ $\in \operatorname{Dom}(F)$, if $\alpha \in \Phi_{i} \leftrightarrow \beta \in \Phi_{i}$ and $\neg \alpha \in \Phi_{i} \leftrightarrow \neg \beta \in \Phi_{i}$ for every $i \in N$, then $\alpha \in F\left(\left(\Phi_{i}\right)_{i \in N}\right) \leftrightarrow \beta \in F\left(\left(\Phi_{i}\right)_{i \in N}\right)$.

Similarly, these two versions are the same if we assume individual completeness, and the strong version is intuitively too strong to be acceptable. Since in the profile even if all judges who abstain on $\alpha$ reject $\beta$, no matter how big portion of these judges is, the strong neutrality requires the group judgment on $\alpha$ and $\beta$ to be the same so long as the set of judges accepting $\alpha$ and the set of judges accepting $\beta$ are the same. The weak version solves the problem.

The last condition is the counterpart of Systematicity, introduced by List and Pettit (2002), which combines independency and neutrality: literals should be treated in an even-handed way by the aggregation function; the group judgment on each literal should depend exclusively on the pattern of individual judgment on that literal.

Strong Literal Systematicity $\left(\mathbf{L S}^{s}\right)$. For every $\alpha, \beta \in \mathcal{P}$ and every profiles $\left(\Phi_{i}\right)_{i \in N},\left(\Phi_{i}^{\prime}\right)_{i \in N} \in \operatorname{Dom}(F)$, if for every $i \in N, \alpha \in \Phi_{i} \leftrightarrow \beta \in \Phi_{i}^{\prime}$, then $\alpha \in F\left(\left(\Phi_{i}\right)_{i \in N}\right) \leftrightarrow \beta \in F\left(\left(\Phi_{i}^{\prime}\right)_{i \in N}\right)$.

Weak Literal Systematicity (LS). For every $\alpha, \beta \in \mathcal{P}$ and every profiles $\left(\Phi_{i}\right)_{i \in N},\left(\Phi_{i}^{\prime}\right)_{i \in N} \in \operatorname{Dom}(F)$, if for every $i \in N, \alpha \in \Phi_{i} \leftrightarrow \beta \in \Phi_{i}^{\prime}$ and $\neg \alpha \in \Phi_{i} \leftrightarrow \neg \beta \in \Phi_{i}^{\prime}$, then $\alpha \in F\left(\left(\Phi_{i}\right)_{i \in N}\right) \leftrightarrow \beta \in F\left(\left(\Phi_{i}^{\prime}\right)_{i \in N}\right)$.

The reason why we reserve Independence, Neutrality and Systematicity to literals alone is based on the consideration that the problem of the doctrinal paradox and the discursive dilemma in Introduction comes from the requirement that the majority rule treats the compound formulas and propositional variables independently. Indeed the principle of compositionality, a fundamental presupposition of the semantics in most contemporary logics, denotes that the propositional variables are more primary than the compound formulas, since the truth of the later is determined by the truth of the former. For instance, in doctrinal paradox, the truth of the conjunctive formula $p \wedge q$ is determined by its constituents $p$ and $q$. In this sense, we may say the judgments on $p$ and $q$ are the reasons to accept $p \wedge q$ or not, while the reason for whether $p$ or $q$ is accepted or not is beyond the expressivity of propositional logic. Of course in more powerful logic such as first-order logic, propositional variables are not primary atoms any more. In that more refined logic, once it presupposes the principle of compositionality, the new building blocks which replace propositional variables are the reasons for accepting propositional variables or not.

In the light of this thought, we take a reason-based perspective and apply the aggregation rule only to primary data whose reasons are beyond the expressivity power of the underlying logics, then use them to generate complex formulas within the underlying logic [23, 26]. Under abstentions, it is the literals instead 
of propositional variables that are primary data, since without completeness, we can not derive that $p$ is rejected from that $p$ is not accepted. It may be possible that $p$ is abstained (neither accepted nor rejected). Therefore, we amount to reserving Independence, Neutrality and Systematicity to literals instead of propositional variables, which makes them more acceptable. For instance, one criticizes Systematicity (the independent part) being used for $p \vee q$, where $p$ denotes "The government can afford a budget deficit", and $q$ "Forbidding smoking should be legalized" on the ground that there are two propositions involved, and that the society should know how each individual feels about either proposition, and not just about their disjunction. There is no similar objection arising when Systematicity applies to either $p$ or $q$ [23].

Another advantage is that this provides a plausible solution for the paradoxes in Introduction. For instance, we may just apply the majority rule to literals and calculate $p, q$ in the group judgment set; then use them to generate $p \wedge q$ in the group judgment set. According to this procedure, the group judgment set is $\{p, q, p \wedge q\}$ which is logically consistent.

In the following we denote Universal Domain, Collective Rationality, Literal Unanimity with Abstentions and Weak Literal Systematicity as UD, CR, LU and LS for short.

\section{The Literal-Based Lexicographic Aggregation Rule}

In this section, we improve the work [21] by proposing an aggregation rule for judgment aggregation with abstentions under voters' hierarchy that works well over a set of logically interconnected formulas instead of a set of literals.

As we have mentioned in the introduction, we assume that there is a hierarchy among all the individuals. In the real-world we can easily see such a hierarchy, for instance, the management structure of an enterprise, a democratic political regime or a community organisation. Members in different ranks may play different roles in collective decision-making.

Definition 1. A hierarchy over the set $N$ of individuals is a strict partial order $<$ over $N$ that satisfies transitivity and asymmetry.

It follows that there is no infinite ascending sequence $i_{1}<i_{2}<i_{3}<\cdots$, where $i_{n} \in N$, which means all hierarchical chains of $N$ must be "up-bounded" with at least one top leader. In this sense, we say $(N,<)$ is well-prioritized.

An aggregation rule determines which propositions are collectively accepted and which ones are collectively rejected. As we denoted, $X_{0}=X \cap \mathcal{P}$ is the set of literals included in the agenda. We first define an aggregate procedure $\mathrm{F}$ for that a literal $\alpha \in X_{0}$ is collectively accepted, denoted by $\alpha \in F\left(\left(\Phi_{i}\right)_{i \in N}\right)$, as follows:

Definition 2. For every $\alpha \in X_{0}$,

$\alpha \in F\left(\left(\Phi_{i}\right)_{i \in N}\right)$ iff $\forall i \in N\left(\neg \alpha \notin \Phi_{i} \vee \exists j \in N\left(i<j \wedge \alpha \in \Phi_{j}\right)\right)$ and $\exists k \in N . \alpha \in \Phi_{k}$ 
Intuitively, this aggregate procedure says that a literal $\alpha$ is accepted by a group if the following two conditions are both satisfied.

(1) for any individual if he rejects $\alpha$, then there is an individual with higher hierarchy accepting $\alpha$; and

(2) there is at least one individual accepting $\alpha$.

We denote the set of collectively accepted literals by $F\left(\left(\Phi_{i}\right)_{i \in N}\right)_{0}$. Based on this concept, we define that any $\varphi \in X$ is collectively accepted as follows:

Definition 3. For any $\varphi \in X$,

$$
\varphi \in F\left(\left(\Phi_{i}\right)_{i \in N}\right) \text { iff } F\left(\left(\Phi_{i}\right)_{i \in N}\right)_{0} \models \varphi
$$

This definition says that a proposition $\varphi$ in the agenda $X$ is collectively accepted if it is a logical consequence of the set of collectively accepted literals.

Similarly, a proposition $\varphi \in X$ is collectively abstained if neither itself nor its negation is collectively accepted. That is,

$$
\varphi \# F\left(\left(\Phi_{i}\right)_{i \in N}\right) \text { iff } \varphi \notin F\left(\left(\Phi_{i}\right)_{i \in N}\right) \text { and } \neg \varphi \notin F\left(\left(\Phi_{i}\right)_{i \in N}\right) .
$$

We call above defined judgment aggregation rule $F$ the literal-based lexicographic rule since we just apply the lexicographic rule to the subset of literals in the agenda.

To demonstrate how this rule works, let us consider the following example.

Example 1. Suppose Ann, Bill and Tom have to make group judgments on three logically connected propositions as follows:

p: There is the elixir of life.

q: People can be immortal.

$p \rightarrow q$ : If there is the elixir of life, then people can be immortal.

Ann thinks $p$ is true and abstains on $q$ and $p \rightarrow q$, Bill rejects $q$ and abstains on $p$ and $p \rightarrow q$, Tom accepts $q$ and $p \rightarrow q$, and abstains on $p$. Their individual judgments are shown as follows:

Table 3.

\begin{tabular}{|r|c|c|c|}
\hline & $\mathrm{p}$ & $\mathrm{q}$ & $\mathrm{p} \rightarrow \mathrm{q}$ \\
\hline Ann & $\mathrm{T}$ & $\#$ & $\#$ \\
Bill & $\#$ & $\mathrm{~F}$ & $\#$ \\
Tom & $\#$ & $T$ & $\mathrm{~T}$ \\
\hline
\end{tabular}


The hierarchy among them is Ann $<$ Bill and Tom $<$ Bill as illustrated below:

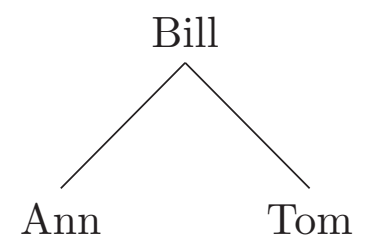

Fig. 1.

Note that individuals with the highest priority are written at the top of the diagram. We next apply the literal-based aggregation rule to generate the collective judgment set. The model of this aggregation situation is as follows:

- $N=\{$ Ann, Bill,Tom $\}$ with Ann $<$ Bill and Tom $<$ Bill;

- $X=\{p, q, p \rightarrow q, \neg p, \neg q, \neg(p \rightarrow q)\}$ and $X_{0}=\{p, q, \neg p, \neg q\}$.

The individual judgment sets for Ann, Bill and Tom are as follows:

- $p \in \Phi_{A n n}, q \# \Phi_{A n n}, p \rightarrow q \# \Phi_{A n n}$;

- $p \# \Phi_{\text {Bill }}, \neg q \in \Phi_{\text {Bill }}, p \rightarrow q \# \Phi_{\text {Bill }}$;

- $p \# \Phi_{T o m}, q \in \Phi_{T o m}, p \rightarrow q \in \Phi_{\text {Tom }}$.

We first calculate the group judgments on the set $X_{0}$ of literals by Definition 2 .

- The collective accepts $p$, since all of them don't reject $p$, i.e., $\forall i \in N\left(\neg p \notin \Phi_{i}\right)$ holds, and Ann accepts $p$, i.e., $\exists j \in N\left(p \in \Phi_{j}\right)$ holds.

- The collective rejects $q$, since Bill with the highest priority rejects $q$, even though Tom accepts $q$.

Then the collective accepts $p$ and rejects $q$, i.e., $F\left(\left(\Phi_{i}\right)_{i \in N}\right)_{0}=\{p, \neg q\}$. And by Definition 3 , the collective rejects $(p \rightarrow q)$, since $\{p, \neg q\} \models \neg(p \rightarrow q)$. Thus, the group judgment set is $\{p, \neg q, \neg(p \rightarrow q)\}$ by the literal-based lexicographic aggregation rule.

We would like to remind that the quasi-lexicographic rule in [21] fails to deal with this situation, since the agenda involves logically interconnected formula $p \rightarrow q$. If we apply that rule to this agenda, we could get an inconsistent aggregate result $\{p, \neg q, p \rightarrow q\}$.

Moreover, the literal-based lexicographic aggregation rule provides a plausible solution to the paradox in Introduction. Let's consider the discursive dilemma. We may take all the possible hierarchy among the three agents into consideration. One boss case: let $1<2<3$ be the hierarchy, then according to this rule the aggregate result is just the individual aggregate set of the first agent $\{p, q, p \rightarrow$ $q\}$, which is consistent. Yet the cost of this case is that the first agent seems to be the dictator for this profile, which is a bit depressing. Two-boss case: let the priority order be $1<3$ and $1<2$, then in the virtue of this rule, they collectively reject $q$ and abstain on $p$ and $p \rightarrow q$. Three-boss (no boss or anonymity) case: according to this rule, $p, q$ and $p \rightarrow q$ are all collectively abstained.

We next investigate the properties of the literal-based lexicographic rule $F$. The first proposition shows that the literal-based lexicographic rule $F$ satisfies above desirable conditions UD, CR, LU and LS. 
Theorem 1. The literal-based lexicographic rule F satisfies conditions UD, CR, $L U$ and $L S$.

Proof. It is straightforward that $F$ satisfies conditions UD according to the definition.

We next show $F$ satisfies CR.

(Logical Closure) For any $\varphi \in X$ and for all $\left\{\Phi_{i}\right\}_{i \in N}$, assume $F\left(\left\{\Phi_{i}\right\}_{i \in N}\right) \models \varphi$. Since $F\left(\left\{\Phi_{i}\right\}_{i \in N}\right)_{0} \models F\left(\left\{\Phi_{i}\right\}_{i \in N}\right)\left(F\left(\left\{\Phi_{i}\right\}_{i \in N}\right)_{0} \models \psi\right.$ for any $\left.\psi \in F\left(\left\{\Phi_{i}\right\}_{i \in N}\right)\right)$, so $F\left(\left\{\Phi_{i}\right\}_{i \in N}\right)_{0} \models \varphi$, so $\varphi \in F\left(\left\{\Phi_{i}\right\}_{i \in N}\right)$ by Definition 3 .

(Consistence) We first show for any $\alpha \in \mathcal{P}, \alpha \in F\left(\left\{\Phi_{i}\right\}_{i \in N}\right)_{0}$ implies $\neg \alpha \notin$ $F\left(\left\{\Phi_{i}\right\}_{i \in N}\right)_{0}$. Suppose for a contradiction that for some $\beta \in \mathcal{P}, \beta \in F\left(\left\{\Phi_{i}\right\}_{i \in N}\right)_{0}$ and $\neg \beta \in F\left(\left\{\Phi_{i}\right\}_{i \in N}\right)_{0}$, then (i) $\forall i \in N\left(\neg \beta \notin \Phi_{i} \vee \exists j \in N\left(i<j \wedge \beta \in \Phi_{j}\right)\right)$ and $\exists k \in N . \beta \in \Phi_{k}$; (ii) $\forall i \in N\left(\beta \notin \Phi_{i} \vee \exists j \in N\left(i<j \wedge \neg \beta \in \Phi_{j}\right)\right)$ and $\exists k \in$ $N . \neg \beta \in \Phi_{k}$. By (i), (ii) we can get an infinite ascending sequence $i_{1}, i_{2}, i_{3}, \cdots$, which is a contradiction with that $(N,<)$ is well-prioritized. Then $F\left(\left\{\Phi_{i}\right\}_{i \in N}\right)_{0}$ is consistent, so by the Definition $3, F\left(\left\{\Phi_{i}\right\}_{i \in N}\right)$ is consistent as well.

For LU, assume for every $\alpha \in \mathcal{P}$, if there is some $V \subseteq N$ such that $V \neq \emptyset$, $\forall i \in V . \alpha \in \Phi_{i}$ and $\forall j \in N \backslash V . \alpha \# \Phi_{j}$, then by Definition $2, \alpha \in F\left(\left\{\Phi_{i}\right\}_{i \in N}\right)$.

For LS, given every $\alpha \in \mathcal{P}$, the individuals accepting $\alpha$ and these rejecting $\alpha$ are the same for every two profiles $\left(\Phi_{i}\right)_{i \in N},\left(\Phi_{i}^{\prime}\right)_{i \in N}$, then the aggregate results of $\alpha$ according to the Definition 2 are the same as well. Yet it is not the case for $\mathrm{LS}^{s}$, for we could construct a counterexample: given a hierarchy on an agent set $\mathrm{N}=\{1,2,3\}$ with $1<2,1<3$. For the profile $\left(\Phi_{i}\right)_{i \in N}$ where $\alpha \in \Phi_{1}, \alpha \# \Phi_{2}$ and $\alpha \# \Phi_{3}$, we have $\alpha \in F\left(\Phi_{i}\right)_{i \in N}$ by $L U$. Let individual 2,3 who abstain on it turn to rejecting $\alpha$, while individual 1 still accepts $\alpha$, we get a different profile $\left(\Phi_{i}^{\prime}\right)_{i \in N}$, where $\alpha \in \Phi_{1}^{\prime}, \neg \alpha \in \Phi_{2}^{\prime}$ and $\neg \alpha \in \Phi_{3}^{\prime}$, but $\alpha \notin F\left(\Phi_{i}^{\prime}\right)_{i \in N}$.

As we expected, $F$ satisfies the four desirable conditions and thus is a feasible aggregation rule for hierarchical groups to generate group judgments. One may be surprised to find that as an 'unfair' aggregation rule, $F$ is non-dictatorial by Proposition 1 . On the other hand, this shows that non-dictatorship is a very weak condition imposed on judgment aggregation functions when abstention is allowable.

Gärdenfors and Dietrich et al have both showed that by giving up completeness, oligarchies instead of dictatorships are obtained [18, 20]. We investigate whether this proposed rule is oligarchic. The definition of an oligarchic rule is given as follows:

Definition 4. An aggregation rule $G$ satisfying $U D$ is a weak oligarchy if there is a non-empty smallest subset $M \subseteq N$ such that for every profile $\left(\Phi_{i}\right)_{i \in N} \in$ $\operatorname{Dom}(G)$,

$$
\bigcap_{i \in M} \Phi_{i} \subseteq G\left(\left(\Phi_{i}\right)_{i \in N}\right)
$$

And an oligarchic rule $G$ is strict if for every profile $\left(\Phi_{i}\right)_{i \in N} \in \operatorname{Dom}(G)$,

$$
\bigcap_{i \in M} \Phi_{i}=G\left(\left(\Phi_{i}\right)_{i \in N}\right) \text {. }
$$

In this case, we call $G$ to be weakly (strictly) oligarchic with respect to $M$. 
That is, an aggregation rule satisfying universal domain is said to be weakly oligarchic if there is a non-empty smallest set $M$ such that for any profile of individual judgment set, the group judgment set contains all the propositions if they are in every member's judgment set of $M$. Furthermore, we say an aggregation rule is strictly oligarchic if for any profile of individual judgment set, the group judgment set is exactly the set of propositions that are in every member's judgment set of $M$. Special cases of weak oligarchic aggregation rules are unanimous $(M=N)$, majority $\left(|M|>\frac{n}{2}\right)$ and dictatorial $(M=\{i\})$ rules. Specifically, the unanimous and dictatorial rules are weakly and strictly oligarchic rules, majority rule is weakly but not strictly oligarchic. However, the literal-based lexicographic rule $F$ is neither weakly oligarchic nor strictly oligarchic. Here is a simple counter-example.

Let the agent set $N=\{1,2\}$ with $<=\emptyset$, the agenda $X=\{p, q, p \rightarrow$ $q, \neg p, \neg q, \neg(p \rightarrow q)\}$ and the set of literals in the agenda $X_{0}=\{p, q, \neg p, \neg q\}$, the individual judgment set for each agent is as follows: $\Phi_{1}=\{p, q, p \rightarrow q\}$, $\Phi_{2}=\{\neg p, \neg q, p \rightarrow q\}$. Then $\Phi_{1} \cap \Phi_{2}=\{p \rightarrow q\}$, but according to the literalbased aggregation rule $F, p \# F\left(\Phi_{1}, \Phi_{2}\right), q \# F\left(\Phi_{1}, \Phi_{2}\right)$ and $p \rightarrow q \# F\left(\Phi_{1}, \Phi_{2}\right)$. Thus, $\Phi_{1} \cap \Phi_{2} \nsubseteq F\left(\Phi_{1}, \Phi_{2}\right)$.

It may be a bit surprising to find that the literal-based aggregation rule is not oligarchic. In fact this does not violates the results in $[18,20]$ since their conditions imposed on the aggregation rules are more strengthened than ours. Specifically, the unanimity and systemacity conditions hold for all formulas while we restrict them to literals. On the other hand, our proposed rule is literalbased, and the compound formulas are dependent on the collective judgments on the literals instead of generating by this rule. That is, if a formula is a logical consequence of the collective literals, then it is in the collective set; otherwise, it is abstained. Therefore, Instead of the whole agenda, we need to consider the oligarchy notion with respect to the set of literals in the agenda on which the literal-based aggregation rule takes effect. This idea leads to a weak concept of an oligarchic aggregation rule as follows:

Definition 5. An aggregation rule $G$ satisfying $U D$ is a weak oligarchy w.r.t $X_{0}$ if there is a non-empty smallest subset $M \subseteq N$ such that for every profile $\left(\Phi_{i}\right)_{i \in N} \in \operatorname{Dom}(G)$,

$$
\left\{\varphi \in X \mid \bigcap_{i \in M} \Phi_{i} \cap X_{0} \models \varphi\right\} \subseteq G\left(\left(\Phi_{i}\right)_{i \in N}\right) .
$$

And an oligarchic rule $G$ is strict w.r.t $X_{0}$ if for every profile $\left(\Phi_{i}\right)_{i \in N} \in \operatorname{Dom}(G)$,

$$
\left\{\varphi \in X \mid \bigcap_{i \in M} \Phi_{i} \cap X_{0} \models \varphi\right\}=G\left(\left(\Phi_{i}\right)_{i \in N}\right) .
$$

Intuitively, an aggregation rule satisfying universal domain is said to be weakly oligarchic w.r.t $X_{0}$ if there is a non-empty smallest set $M$ such that for any 
profile of individual judgment set, the group judgment set contains all the consequences of literals that are in every member's judgment set of $M$. Similarly, an aggregation rule is strictly oligarchic w.r.t $X_{0}$ if for any profile of individual judgment set, the group judgment set is exactly the set of consequences of the literals that are in every member's judgment set of $M$.

We have the following proposition saying the literal-based lexicographic rule $F$ is weakly oligarchic w.r.t $X_{0}$, but not strictly oligarchic w.r.t $X_{0}$.

Proposition 2. The literal-based lexicographic rule $F$ is weakly oligarchic w.r.t $X_{0}$, but not strictly oligarchic w.r.t $X_{0}$.

Proof. Suppose $F$ satisfies universal domain, it suffices to find a non-empty smallest set $M \subseteq N$, such that for every profile of individual judgment sets $\left(\Phi_{i}\right)_{i \in N}$, every $\varphi \in X$, if $\bigcap_{i \in M} \Phi_{i} \cap X_{0} \models \varphi$, then $\varphi \in F\left(\left(\Phi_{i}\right)_{i \in N}\right)$. Let $O=$ $\operatorname{Max}_{\geq}(N)=\{i \in N: \nexists j \in N . i<j\}$. Since $(N,<)$ is well-prioritized and $|N| \geq$ 2 , so $O$ must exist and be non-empty. Suppose for every profile of individual judgment sets $\left(\Phi_{i}\right)_{i \in N}$, every $i \in N$ and for all $\alpha \in X_{0}$, if $\alpha \in \bigcap_{i \in O} \Phi_{i}$, then according to Definition 2, $\alpha \in F\left(\left(\Phi_{i}\right)_{i \in N}\right)$. Then $\bigcap_{i \in O} \Phi_{i} \cap X_{0} \subseteq F\left(\left(\Phi_{i}\right)_{i \in N}\right)_{0}$. Since $\bigcap_{i \in O} \Phi_{i} \cap X_{0} \models \varphi$, so $F\left(\left(\Phi_{i}\right)_{i \in N}\right)_{0} \models \varphi$, so $\varphi \in F\left(\left(\Phi_{i}\right)_{i \in N}\right)$ by Definition 3 .

We next show $O$ is the smallest one with $\left\{\varphi \in X \mid \bigcap_{i \in O} \Phi_{i} \cap X_{0} \models \varphi\right\} \subseteq$ $F\left(\left(\Phi_{i}\right)_{i \in N}\right)$. Suppose not, then there is some $A \subseteq N$ such that $A \subset O$ and $\left\{\varphi \in X \mid \bigcap_{i \in A} \Phi_{i} \cap X_{0} \models \varphi\right\} \subseteq F\left(\left(\Phi_{i}\right)_{i \in N}\right)$, then there is some $a \in N$ such that $a \in O$ but $a \notin A$. Take some $\beta \in X_{0}$ and define $\beta \in \Phi_{i}$ for every $i \in N \backslash\{a\}$ and $\neg \beta \in \Phi_{a}$, then by Definition $2, \beta \notin F\left(\left(\Phi_{i}\right)_{i \in N}\right)$, but $\beta \in\left\{\varphi \in X \mid \bigcap_{i \in A} \Phi_{i} \cap\right.$ $\left.X_{0} \models \varphi\right\}$, contradicting with assumption. Thus, $O$ is just the required $\mathrm{M}$.

It's easy to construct a profile such that $\bigcap_{i \in M} \Phi_{i} \cap X_{0} \nsupseteq F\left(\left(\Phi_{i}\right)_{i \in N}\right)$ by LU. Take $\alpha \in X_{0}$ and define $\alpha \# \Phi_{a}$ for some $a \in M$ and $\alpha \in \Phi_{x}$ for every $x \in N /\{a\}$. By literal unanimity with abstentions, $\alpha \in F\left(\left(\Phi_{i}\right)_{i \in N}\right)$, but $\alpha \notin \bigcap_{i \in M} \Phi_{i} \cap X_{0}$. Thus $F$ is not strictly oligarchic w.r.t $X_{0}$.

On the one hand the literal-based lexicographic aggregation rule $F$ satisfies all the desirable conditions given in Section 3; on the other hand, it is only oligarchic with respect to the subset of literals in the agenda not over the whole set of agenda due to its literal-base characteristics.

The last proposition of this section shows under which conditions two literalbased lexicographic rules are identical. We first introduce a helpful notation. For any $i \in N$ and any $\varphi, \psi \in X$, the individual $i$ makes the same judgment on $\varphi$ and $\psi$ is denoted by $\left.\left.\Phi_{i}\right|_{\{\varphi\}} \Leftrightarrow \Phi_{i}\right|_{\{\psi\}}$, that is $\left.\left.\Phi_{i}\right|_{\{\varphi\}} \Leftrightarrow \Phi_{i}\right|_{\{\psi\}}$ is equivalent to $\left(\varphi \in \Phi_{i}\right.$ iff $\left.\psi \in \Phi_{i}\right)$ and $\left(\neg \varphi \in \Phi_{i}\right.$ iff $\left.\neg \psi \in \Phi_{i}\right)$.

Proposition 3. Let $\{p\} \subseteq X \cap \Phi_{0}$, and $f_{1}$, $f_{2}$ be two literal-based lexicographic rules. If for all profiles of individual judgment sets $\left(\Phi_{i}\right)_{i \in N},\left.f_{1}\left(\left(\Phi_{i}\right)_{i \in N}\right)\right|_{\{p\}}$ $\left.\Leftrightarrow f_{2}\left(\left(\Phi_{i}\right)_{i \in N}\right)\right|_{\{p\}}$, then for all $\varphi \in X,\left.\left.f_{1}\left(\left(\Phi_{i}\right)_{i \in N}\right)\right|_{\{\varphi\}} \Leftrightarrow f_{2}\left(\left(\Phi_{i}\right)_{i \in N}\right)\right|_{\{\varphi\}}$. 
Proof. Take any $q \in X \cap \Phi_{0}$. Define the profile $\left(\Phi_{i}^{\prime}\right)_{i \in N}$ in terms of $\left(\Phi_{i}\right)_{i \in N}$ as follows: for all $i \in N, \Phi_{i}^{\prime}=\Phi_{i}$ except at $p$ where $\left.\left.\Phi_{i}^{\prime}\right|_{\{p\}} \Leftrightarrow \Phi_{i}\right|_{\{q\}}$. Then

$$
\begin{aligned}
\left.f_{1}\left(\left(\Phi_{i}\right)_{i \in N}\right)\right|_{\{q\}} & \Leftrightarrow f_{1}\left(\left(\left.\Phi_{i}\right|_{\{q\}}\right)_{i \in N}\right) \text { by LI } \\
& \Leftrightarrow f_{1}\left(\left(\left.\Phi_{i}^{\prime}\right|_{\{p\}}\right)_{i \in N}\right) \text { by LS } \\
& \left.\Leftrightarrow f_{1}\left(\left(\Phi_{i}^{\prime}\right)_{i \in N}\right)\right|_{\{p\}} \text { by LI } \\
& \left.\Leftrightarrow f_{2}\left(\left(\Phi_{i}^{\prime}\right)_{i \in N}\right)\right|_{\{p\}} \text { by hypothesis } \\
& \Leftrightarrow f_{2}\left(\left(\left.\Phi_{i}^{\prime}\right|_{\{p\}}\right)_{i \in N}\right) \text { by LI } \\
& \Leftrightarrow f_{2}\left(\left(\left.\Phi_{i}\right|_{\{q\}}\right)_{i \in N}\right) \text { by LS } \\
& \left.\Leftrightarrow f_{2}\left(\left(\Phi_{i}\right)_{i \in N}\right)\right|_{\{q\}} \text { by LI. }
\end{aligned}
$$

It is clear that $f_{1}\left(\left(\Phi_{i}\right)_{i \in N}\right) \cap \Phi_{0}=f_{2}\left(\left(\Phi_{i}\right)_{i \in N}\right) \cap \Phi_{0}$.

Hence, $f_{1}\left(\left(\Phi_{i}\right)_{i \in N}\right)=f_{2}\left(\left(\Phi_{i}\right)_{i \in N}\right)$.

This proposition says that if two literal-based lexicographic rules make a same judgment (acceptance, rejection and abstention) on a propositional variable in the agenda, then they make the same judgment on all the formulas in the agenda. In the other word, a literal-based lexicographic rule is determined by its responses to all the possible judgments (acceptance, rejection and abstention) on a fixed propositional variable in the agenda. This property paves the way to show a characterization result of the literal-based lexicographic rule. We leave this work for future.

\section{Conclusion}

In this paper, we have proposed a literal-based lexicographic rule which extends the set of agenda from a set of literals to a set of logically interconnected formulas [21] and further investigated the properties of this rule. It turns out that this proposed rule is neither dictatorial nor oligarchic in the standard sense. The reason for non-dictatorship is that, as proved in Proposition 1, we may take advantage of the abstention to show all individuals fail to play a role in collective decision-making on a particular proposition when they abstain from that proposition. This indicates that under the provision of abstentions, non-dictatorship is a rather weak condition imposed on the judgment aggregation rule. The reason for non-oligarchy is due to its literal-based characteristics. In fact, we may regard this proposed rule as a special case of premiss-based rule [27] where the set of premisses is the subset of literals in the agenda.

There are many directions for future investigations. Firstly, as a special kind of lexicographic rule, it is interesting to investigate a representation result for our proposed aggregation rule. The lexicographic rule has been extensively studies in preference aggregation, and [28] has proved that lexicographic rule is the only way of combining preference relations satisfying five natural conditions which are very close to Arrow's conditions. We expect to obtain a similar characterization result for our proposed rule. This work is under way;

Secondly, under the provision of abstentions, we have amended the commonly desirable conditions. It is natural to investigate some possibility results with 
respect to these amending conditions. In terms of the rationality requirements, this means that we drop the completeness condition. Comparing with the impossibility results, we get a possibility result which can be stated as: there are non-dictatorial aggregation rules satisfying universal domain, collective rationality, literal unanimity with abstentions and weak literal systematicity, which seems positive news to the result in [23]. However, it is not the case since we do not assume completeness at both individual and collective levels, which means that a judge can abstain from a proposition and the group judgment on a proposition can be undetermined.

In addition, with abstentions, the dictatorship in judgment aggregation can also vary in degrees [29]. It is highly interesting to investigate the possibility scope between rationality, dictatorship under a set of plausible conditions. Some work has been done in this direction [30].

Last but not least, since the universal and perfect aggregation rule does not exist, a plausible way is to boil down aggregation problems to three-sided matching questions between specific so-called degenerate rules, specific groups and specific agendas., which is a promising direction [22].

\section{References}

1. Dietrich, F., List, C.: Arrow's theorem in judgment aggregation. Social Choice and Welfare 29(1), 19-33 (2007)

2. Mongin, P.: Consistent bayesian aggregation. Journal of Economic Theory 66(2), 313-351 (1995)

3. Pigozzi, G.: Belief merging and the discursive dilemma: an argument-based account to paradoxes of judgment aggregation. Synthese 152(2), 285-298 (2006)

4. Wilson, R.: On the theory of aggregation. Journal of Economic Theory 10(1), 89-99 (1975)

5. Konieczny, S., Pérez, R.P.: Merging information under constraints: a logical framework. Journal of Logic and Computation 12(5), 773-808 (2002)

6. Kornhauser, L.A., Sager, L.G.: Unpacking the court. The Yale Law Journal 96(1), 82-117 (1986)

7. List, C.: The theory of judgment aggregation: an introductory review. Synthese 187(1), 179-207 (2012)

8. Pettit, P.: Deliberative democracy and the discursive dilemma. Philosophical Issues 11(1), 268-299 (2001)

9. Pivato, M.: The discursive dilemma and probabilistic judgement aggregation. Munich Personal RePEc Archive (2008)

10. Arrow, K.J.: A difficulty in the concept of social welfare. Journal of Political Economy 58, 328-346 (1950)

11. List, C., Pettit, P.: Aggregating sets of judgments: An impossibility result. Economics and Philosophy 18(01), 89-110 (2002)

12. Dietrich, F.: Judgment aggregation (im)possibility theorems. Journal of Economic Theory 126(1), 286-298 (2006)

13. List, C., Pettit, P.: Aggregating sets of judgments: Two impossibility results compared. Synthese 140(1-2), 207-235 (2004)

14. Mongin, P.: Factoring out the impossibility of logical aggregation. Journal of Economic Theory 141(1), 100-113 (2008) 
15. Pauly, M., Van Hees, M.: Logical constraints on judgement aggregation. Journal of Philosophical logic 35(6), 569-585 (2006)

16. Van Hees, M.: The limits of epistemic democracy. Social Choice and Welfare 28(4), 649-666 (2007)

17. Dietrich, F., List, C.: Judgment aggregation with consistency alone. London School of Economics, Government Department, London, UK (2007)

18. Dietrich, F., List, C.: Judgment aggregation without full rationality. Social Choice and Welfare 31(1), 15-39 (2008)

19. Dokow, E., Holzman, R.: Aggregation of binary evaluations with abstentions. Journal of Economic Theory 145(2), 544-561 (2010)

20. Gärdenfors, P.: A representation theorem for voting with logical consequences. Economics and Philosophy 22(2), 181-190 (2006)

21. Jiang, G., Zhang, D., Tang, X.: Judgment aggregation with abstentions: A hierarchical approach. In: Grossi, D., Roy, O., Huang, H. (eds.) LORI. LNCS, vol. 8196, pp. 321-325. Springer, Heidelberg (2013)

22. Dietrich, F., List, C.: A liberal paradox for judgment aggregation. Social Choice and Welfare 31(1), 59-78 (2008)

23. Mongin, P.: Factoring out the impossibility of logical aggregation. Journal of Economic Theory 141(1), 100-113 (2008)

24. Dietrich, F.: A generalised model of judgment aggregation. Social Choice and Welfare 28(4), 529-565 (2007)

25. Arrow, K.J.: Social Choice and Individual Values. Cowles Foundation Monographs. Wiley, New York $(1953,1964)$

26. Nehring, K., Puppe, C.: Consistent judgement aggregation: the truth-functional case. Social Choice and Welfare 31(1), 41-57 (2008)

27. Dietrich, F., Mongin, P.: The premiss-based approach to judgment aggregation. Journal of Economic Theory 145(2), 562-582 (2010)

28. Andréka, H., Ryan, M., Schobbens, P.Y.: Operators and laws for combining preference relations. Journal of Logic and Computation 12(1), 13-53 (2002)

29. Rossi, F., Venable, K.B., Walsh, T.: Aggregating preferences cannot be fair. Intelligenza Artificiale 2(1), 30-38 (2005)

30. Dietrich, F., List, C.: Propositionwise judgment aggregation: the general case. Social Choice and Welfare 40(4), 1067-1095 (2013) 